\title{
Various Expression and Clinical Significance of Checkpoint Molecules in Advanced Osteosarcoma: Possibilities for Novel Immunotherapy
}

Lu Xie

Peking University People's Hospital

Chenglong Chen

Peking University People's Hospital

Xin Liang

Peking University People's Hospital

Jie Xu

Peking University People's Hospital

Xin Sun

Peking University People's Hospital

Kunkun Sun

Peking University People's Hospital

Rongli Yang

Peking University People's Hospital

Xiaodong Tang

Peking University People's Hospital

Wei Guo ( $\nabla$ bonetumor@163.com )

Peking University People's Hospital

\section{Research Article}

Keywords: Osteosarcoma, Checkpoint molecules, Coexpression, Immunotherapy, Prognosis

Posted Date: September 8th, 2021

DOI: https://doi.org/10.21203/rs.3.rs-846254/v1

License: (c) (i) This work is licensed under a Creative Commons Attribution 4.0 International License.

Read Full License 


\section{Expression and clinical significance of various checkpoint molecules}

\section{2 in advanced osteosarcoma: possibilities for novel immunotherapy}

3 Lu Xie ${ }^{1 \dagger}$, Chenglong $\mathrm{Chen}^{1 \dagger}$, Xin Liang ${ }^{1}$, Jie $\mathrm{Xu}^{1}$, Xin $\mathrm{Sun}^{1}$, Kunkun Sun ${ }^{2}$, Rongli

4 Yang $^{1}$, Xiaodong Tang ${ }^{1}$ and Wei Guo ${ }^{1 *}$

$5 \quad{ }^{1}$ Musculoskeletal Tumor Center, Peking University People's Hospital, Beijing 100044,

6 China

$7 \quad{ }^{2}$ Pathology Department, Peking University People's Hospital, Beijing 100044, China

$8 \quad{ }^{*}$ Correspondence: bonetumor@163.com

$9 \quad{ }^{\dagger} \mathrm{Lu}$ Xie and Chenglong Chen are co-first authors

\section{Abstract}

11 Background: The fact that studies on anti-programmed cell death 1 (PD-1) or its 12 relevant ligand 1 (PD-L1) have yielded such few responses greatly decreases the 13 confidence in immunotherapy with checkpoint inhibitors for advanced osteosarcoma.

14 We examined 35 advanced osteosarcoma specimens, characterized the expression of 15 various checkpoint molecules with immunohistochemistry and analyzed the 16 relationship of the expression of these checkpoint molecules with patients' clinical 17 courses.

18 Methods: Immunohistochemistry for B7-H3, CD47, PD-L1, TIM3, TGF- $\beta$, CXCR 4, 19 CD27, IDO1, KIRs and SDF-1 was performed on 35 resected advanced osteosarcoma 20 specimens. Associations between the marker levels and clinicopathological variables 21 and survival were evaluated.

22 Results: The positive rates of B7-H3, CD47, PD-L1, TIM3, and TGF- $\beta$ expression in 23 this sample of 35 heavily treated osteosarcomas were $29 \%(10 / 35), 15 \%(5 / 35), 9 \%$ $24(3 / 35), 6 \%(2 / 35)$ and $6 \%(2 / 35)$, respectively, and diverse staining intensities were 
25 observed. Among these advanced patients, 15/35 (43\%) had positive checkpoint expression, of whom 33\% (5/15) showed evidence of the coexpression of more than 1 checkpoint molecule. We did not find any obvious correlation with clinicopathological characteristics and the positive expression of these molecules; however, for this small sample, a tendency for benefiting from anti-PD-1 therapy was observed in patients with positive B7-H3 expression $(P=0.057)$.

31 Conclusions: Our study first reported that only a small subset of progressive osteosarcomas expressed tumor immune-associated checkpoint molecules. Those osteosarcomas that had ever been responsive to anti-PD-1 therapy usually had evidence of the coexpression of multiple checkpoint molecules.

35 Keywords: Osteosarcoma, Checkpoint molecules, Coexpression, Immunotherapy, Prognosis

\section{Background}

Osteosarcomas are bone-forming tumors characterized by the presence of an extracellular osteoid matrix produced by cancer cells and associated with a very complex local environment including bone cells, blood vessels, stromal cells and

41 immune infiltrates [1]. The peak incidence of osteosarcoma occurs during the adolescent growth spurt, which suggests that bone growth and pubertal hormones are important in the etiology of the disease [2]. Although modern multiagent, dose-intensive chemotherapy (in conjunction with surgery) achieves a 5-year event-free survival (EFS) of approximately $60-71 \%$ in extremity localized, nonmetastatic disease $[3,4]$, the prognosis for relapsed or refractory disease that progresses despite chemotherapy remains dismal after decades of clinical trials for new agents [5]. Recently, the remarkable results achieved with the advent of cancer immunotherapies and checkpoint inhibitors have revolutionized the field of oncology 
by putting the host immune response under the spotlight as a target for anticancer

51 therapeutic interventions [6]. Nivolumab [7], ipilimumab [7], pembrolizumab [8, 9] and camrelizumab (SHR-1210) [10] have all been studied in patients with advanced disease either alone or in combination. However, only a limited number of patients have derived meaningful clinical benefits, with no statistical advantage for the whole population $[7,8,10,11]$. Challenges remaining on the path forward include the identification of the most suitable checkpoint and immunotherapy, the prevention of paradoxical or hyperprogressive disease and the exploration of predictive biomarkers for more personalized immunotherapies for osteosarcoma patients [12, 13]. their microenvironment through the secretion of various cytokines, chemokines, and

61 other factors, is complex and continuously evolving [14]. The T cell receptor (TCR) starts the signaling cascade upon its interaction with peptide antigen in the context of the major histocompatibility complex (MHC), but optimal activation of naive T cells depends on a costimulatory signal through CD28 [15]. Additional interactions between ligands and activating or inhibitory receptors are crucial for further regulating $\mathrm{T}$ cell activation and tolerance. Therapeutics targeting these and other pathways are in various stages of clinical development [6]. Osteosarcoma usually acts in an immune rheostat or "immunostat" condition [16]. It is suspected that upregulated immunoinhibitory pathways other than programmed cell death 1 or its relevant ligand 1 (PD-1/PD-L1) might dampen or arrest the antitumor immune

71 response in osteosarcoma [17]. It was recently found in other tumors that combining

72 immunological agents may improve response rates and the duration of response by 73 stimulating antitumor immunological memory $[6,14]$.

74 However, literature on the expression of different checkpoint molecules in 
75 advanced osteosarcoma is scarce [18-23]. Mochizuki et al. [18] explored the 76 expression of various checkpoint molecules and tumor-infiltrating lymphocytes (TILs) 77 with immunohistochemistry in common pediatric solid tumors, and among 12 78 untreated osteosarcoma specimens, $100 \%$ expressed moderate to high levels of 79 herpesvirus entry mediator (HVEM) on the tumor. TILs were detected in all tumor 80 samples except one osteosarcoma sample. Piperdi et al. [23] suggested that CD47 was 81 expressed in $87.7 \%$ of specimens, with $28.4 \%, 27.2 \%$, and $32.1 \%$ demonstrating high, 82 intermediate, and low expression, respectively, in a tissue microarray (TMA) of 81 83 osteosarcoma specimens.

84 In this study, we evaluated the expression of B7-H3, CD47, PD-L1, TIM3, TGF- $\beta$, 85 CXCR 4, CD27, IDO1, KIRs and SDF-1 in 35 advanced osteosarcoma specimens 86 with immunohistochemistry, and we analyzed the associations between these marker 87 levels and clinicopathological variables and survival.

88 Methods

\section{Patients and samples}

90 This study was approved by the Institutional Review Board of Peking University

91 People's Hospital. The samples were collected from the Musculoskeletal Tumor 92 Center of Peking University People's Hospital between August 2017 and March 2020.

93 Written informed consent was obtained from all patients before using their specimens 94 previously stored in the pathological department specimen repository. Table 1 95 summarizes the demographic information of the patients examined in this study. Each 96 sample was pathologically diagnosed as high-grade osteosarcoma by using 97 hematoxylin and eosin (H\&E) staining as well as several required 98 immunohistochemical (IHC) stains. For the current study, two senior pathologists 
99 (SDH and SKK) further confirmed the results of the tissue samples with new HE

100 staining.

102 Clinical follow-up was continuously performed every two months until the death of

103 the patients. The hospital records, laboratory examination results and imaging results

104 of all patients were retrieved and reviewed.

\section{Procedure for immunohistochemistry}

IHC analyses were performed using the avidin-biotin complex (ABC) method [21]. A significant proportion of specimens were decalcified: $87 \%(20 / 23)$ of resected musculoskeletal specimens and 28\% (2/7) of metastasectomy specimens of the lung. Tumor tissue sections ( $5 \mu \mathrm{m}$ thick) were deparaffinized in xylene and rehydrated in a graded series of ethanols. Sections were incubated for $15 \mathrm{~min}$ with $1.0 \%$ hydrogen peroxide $\left(\mathrm{H}_{2} \mathrm{O}_{2}\right)$ to block endogenous peroxidases; rinsed in phosphate-buffered saline (PBS); incubated in $10 \mathrm{mM}$ sodium citrate buffer, $\mathrm{pH}$ 6.0, for $10 \mathrm{~min}$, with an interval of $1 \mathrm{~min}$ and another heating step of $5 \mathrm{~min}$; incubated for $20 \mathrm{~min}$ in normal goat serum (Vector Laboratories, Burlingame, CA) [20] to block nonspecific binding of antibodies; and subjected to antigen retrieval according to the optimal protocol for each primary antibody (Additional file 1: Table S1).

Sections were incubated overnight at $4^{\circ} \mathrm{C}$ with primary antibodies, rinsed in PBS, incubated for 30 min with biotinylated anti-rabbit or anti-mouse goat IgG (Vector Laboratories) as secondary antibodies, rinsed in PBS, incubated for $30 \mathrm{~min}$ with components from a VECTASTAINVR EliteVR ABC KIT (Vector Laboratories), and treated with 3,3'-diaminobenzidine tetrahydrochloride and $\mathrm{H}_{2} \mathrm{O}_{2}$ solution to allow color development. Finally, all slides were rinsed with water, counterstained with hematoxylin, dehydrated in a graded series of ethanols, and cleared in xylene; 


\section{IHC scoring}

126 The immunostaining was interpreted by a pulmonary pathologist (SKK and DY) as 127 negative ( $0 \%$ tumor cell staining) or positive. First, the two pathologists individually 128 assigned IHC scores for all sections in the present study. In this process, the two 129 pathologists were blinded. Then, the two pathologists worked together to reexamine

130 the sections that were assigned different scores and discussed the discrepancies. Then,

131 the pathologists provided final values after reaching an agreement.

132 In this study, only cell surface expression was evaluated for each checkpoint 133 molecule, and we evaluated the percentage of tumor cells with any expression 134 regardless of the intensity of staining [18]. To be considered positive, staining had to 135 be membranous and circumferential.

136 On the tumor cells: A semiquantitative scoring system $(0-<1 \%=$ negative, recorded

137 as $\mathrm{N} ; 1-<5 \%=$ low, recorded as $\mathrm{L} ; 5-<50 \%=$ moderate, recorded as $\mathrm{M}$; and $\geq 50 \%=$ 138 high, recorded as $\mathrm{H}$ ) was applied for the evaluation of immune checkpoint ligand 139 expression [16].

140 On the tumor-infiltrating lymphocytes: Tumor-infiltrating lymphocytes (TILs) were

141 lymphocytes that had infiltrated tumor tissues, and TILs were subjectively identified 142 microscopically by two pathologists individually. A semiquantitative scoring system $143(0-<1 \%=$ negative, recorded as $\mathrm{N} ; 1-<5 \%=$ low, recorded as $\mathrm{L} ; 5-<50 \%=$ moderate, 144 recorded as $\mathrm{M}$; and $\geq 50 \%=$ high, recorded as $\mathrm{H}$ ) was applied for the evaluation of 145 immune checkpoint receptor expression on the surface of TILs [15].

\section{Statistical analysis}

147 All values are expressed as the mean \pm standard error of the mean (SEM). Statistical 
149 Jolla, California, USA) statistical package and R language (V.3.6.1). Interdependence

150 between staining and clinical data was calculated using the $\chi^{2}$ displayed by 151 cross-tables. Student's $t$-test was used for the comparison of data between two groups.

152 The Cox proportional hazards regression model and Kaplan-Meier plots were 153 generated by the survival package, and the log-rank test was used to compare survival 154 curves between different groups. Statistical significance was defined as a $P<0.05$.

\section{Results}

\section{Patient clinicopathological characteristics and outcome}

157 The clinicopathological characteristics of the patients included in the study are

158 summarized in Table 1. All these patients were in a metastatic or refractory state, and 159 their disease had progressed upon first-line chemotherapy [24, 25]. The median age at 160 diagnosis was 14 (interquartile range (IQR), 6, 22) years. The male-to-female ratio 161 was approximately 4:3. Among this population, 30/35 (85.7\%) had conventional 162 osteosarcoma, while $29 / 35(82.9 \%)$ of the primary tumors were located at the 163 extremities. Twenty-five of the 35 specimens $(71.4 \%)$ were resected from 164 musculoskeletal lesions, $7 / 35(20.0 \%)$ were resected from pulmonary metastatic 165 lesions, and 3/35 (8.6\%) were resected from osteosarcoma lymph node metastases.

166 We usually conducted our first-line chemotherapy following the Peking University 167 People's Hospital-Osteosarcoma (PKUPH-OS 02) regimen (Additional file 3: Fig. S1) 168 [24, 25], which included high-dose methotrexate, doxorubicin, cisplatin, and

169 ifosfamide. Our second-line chemotherapy was usually ifosfamide $\left(1.8 \mathrm{~g} / \mathrm{m}^{2} / \mathrm{d} \mathrm{d} 1-5\right)$ 170 and etoposide $\left(100 \mathrm{mg} / \mathrm{m}^{2} / \mathrm{d} \mathrm{d} 1-5 \mathrm{Q}_{3} \mathrm{w}\right)$, while our third-line systemic therapy was 171 usually antiangiogenic tyrosine kinase inhibitor (TKI)-based therapy, such as apatinib 
172 and regorafenib [24, 25]. For this group of patients, 14/35 (40\%) had been confirmed

173 to be refractory to first- and second-line chemotherapy, while 4/35 (11.4\%) had been

174 progressive upon TKI therapy. We tried anti-PD-1 antibodies in combination therapy

175 in some of our patients, but in clinical evaluations, we found confirmed efficacy of

176 this treatment in only 4/35 (11.4\%) patients, with progression-free survival (PFS) of

177 more than 1 year, while this treatment was firmly believed to be invalid in $9 / 35$

$178(25.7 \%)$ of patients. A total of 35 patients (83.3\%) were followed up for an average of

17934.7 months, which ranged from 14.7 to 110.1 months. At the time of the last

180 follow-up, 4 deaths had occurred among the 35 patients.

181 Checkpoint ligands expression on advanced osteosarcoma

182 First, we examined the expression of immune checkpoint ligands on tumor cells. As

183 shown in Fig. 1, the patterns of PD-L1 staining were predominantly membranous.

184 B7-H3 was also detected to be the most commonly expressed checkpoint and was

185 mainly expressed on the cell membrane of tumor cells. In some studies, PD-L1 was

186 also observed in vascular endothelial cells and tumor-infiltrating immune cells.

187 However, in this study, according to the specifications of the B7-H3 antibody,

188 pathologists agreed to judge the expression as negative if tumor cells and background

189 were all stained positive. As shown in Tables 1 and 2, in this sample of tumors, only 9\%

190 (3/35) were PD-L1, 6\% (2/35) were TGF $\beta, 15 \%$ (5/35) were CD47, and 29\% (10/35)

191 were B7-H3 positive; these results are inconsistent with those of a previous study. No

192 CXCR-4 or KIR expression was observed in these heavily treated osteosarcoma

193 samples. Twenty-six percent of osteosarcomas moderate to high expression levels of

194 B7-H3 on their surface, while 3\% had only low expression levels (Table 2). For CD47,

195 only $9 \%$ of osteosarcomas expressed moderate to high levels, while $6 \%$ had low

196 staining intensity. We noticed that $14 \%(5 / 35)$ of our patients showed evidence of the 
coexpression of several checkpoint molecules, and we compared the prognoses of

198

199

201 for this small sample, a tendency for benefiting from anti-PD-1 therapy was observed

202 for patients with positive B7-H3 expression $(P=0.057)$ (Table 1).

211 pathologists. TIM3, a receptor within the subfamily of TIM (T

212 cell-immunoglobulin-mucin domain) proteins, is another $\mathrm{T}$ cell-expressed $\mathrm{IgSF}$

213 protein of significant interest. Multiple TIM3 ligands have been described, including 214 phosphatidylserine, galectin 9 (GAL9), high mobility group protein B1 (HMGB1) and 215 carcinoembryonic antigen-related cell adhesion molecule (CEACAM1). Because the 216 blocking characteristics of therapeutic TIM3-specific antibodies have not been 217 completely described, their mechanism of action is poorly understood, and we did not 218 further explore ligand expression. Interestingly, both of these patients who had TIM3 219 expression also showed evidence of the coexpression of PD-L1 at low intensity levels, 220 and one even had moderate-intensity expression of B7-H3. 
221 Clinical significance of $\mathbf{B} 7$ family protein coexpression in selected osteosarcoma patients

223 We further analyzed the relationship between these high-risk osteosarcoma patients' 224 clinical prognoses and their B7 family ligand/receptor expression. No obvious 225 expression of these molecules and PFS (for chemotherapy resistance) and overall 226 survival $(\mathrm{OS})$ had an inverse association $(P$ all $>0.05)$ (shown in Table 3$)$. However, 227 in this small subset of the study population, we noticed that females and telangiectatic 228 subtypes of osteosarcoma had a tendency for poorer PFS or OS, respectively, which 229 was not in accordance with the long-term follow-up results of European and American 230 Osteosarcoma Study-1 (EURAMOS-1). One of the 5 patients who showed evidence 231 of the coexpression of more than one checkpoint molecule had high levels of B7-H3, 232 CD47 and TGF $\beta$ but was negative for PD-L1 (Fig. 1a-d). He was diagnosed in 233 November 2015 with proximal femoral osteosarcoma (Fig. 1e), which progressed and 234 became chemotherapy-resistant (including adriamycin, cisplatin, high-dose 235 methotrexate and ifosfamide) in August 2017. In 2017 (3 years ago), he had shown 236 evidence of a high level of PD-L1 expression and had a tumor proportion score (TPS) 237 of $50 \%$ (Fig. 1f) in his amputation specimen. He had been in stable disease by 238 combination therapy of pembrolizumab and cabozantinib for one year (2017-2018) 239 and then single pembrolizumab for more than one year (from 2018 to 2020). However, 240 the tumor progressed again in September 2019 in his ipsilateral inguinal lymph nodes, 241 and we performed lesion resection again with IHC staining in the current study. The 242 newly resected lesion was identified as undifferentiated pleomorphic sarcoma (UPS) 243 (Fig. 1g) by our pathologists.

\section{Discussion}


246 According to limited publications [10, 13, 16, 18-23], the current study reported the

247 lowest rate of positive checkpoint molecule expression in advanced osteosarcoma, 248 with B7-H3, CD47, PD-L1, TIM3 and TGF $\beta$ levels as low as 29\%, 15\%, 9\%, 6\% and $2496 \%$, respectively. Pinto et al. [26] also described childhood cancers in which PD-L1 250 was detected in the majority of patients, including those with Ewing sarcoma (65\%), 251 neuroblastoma (77\%), and osteosarcoma (80\%). Koirala et al. [13] showed that 252 patients with PD-L1 expression in osteosarcomas were significantly associated with 253 worse 5-year EFS than patients without PD-L1 expression in osteosarcomas $(25.0 \%$ 254 vs. $69.4 \%, P=0.014)$. We conducted a phase II trial for apatinib plus camrelizumab 255 in patients with advanced osteosarcoma, and those who were positive for PD-L1 (with 256 a PD-L1-positive rate of 21.4\%) seemed to benefit from combination therapy more 257 and have a longer PFS [10]. The reported positivity of PD-L1 in osteosarcoma seems 258 to be variable (Additional file 1: Table S2). The different anti-PD-L1 antibodies used 259 in each study differ in their targeted epitope, and binding affinity may be one of the 260 major reasons for the differences [8-10, 12, 13, 18, 20, 26]. For the other checkpoints, 261 Mochizuki et al. [18] reported that 100\% of osteosarcomas expressed HVEM, and 262 moderate to high levels of GAL9, which was a ligand for TIM3) were observed in $36 \%$ 263 of osteosarcomas, while Piperdi et al. [23] suggested that CD47 was expressed in the 264 TMA of $87.7 \%$ of 81 osteosarcoma specimens.

265 However, this might just be the real situation for heavily treated advanced 266 osteosarcomas based on the very few immune-responsive cases in the past decades of 267 trials for advanced osteosarcoma [7-10]. In this study, we demonstrated the 268 coexpression of checkpoint molecules in $35.7 \%(5 / 14)$ of our patients, usually the 269 coexpression of B7-H3 and CD47 (in 4 patients) or TIM3 and PD-L1 (in 2 patients). 270 False-negative interpretation might still exist, although both of the pathologists 
strictly followed the protocols and if any costaining for tumor cells and TILs was

272 present, they discussed the findings with each other or even a third pathologist to

273 identify whether this was background staining. However, extensive background

274 staining was eventually affirmed and concluded to be negative (shown in Figs. 3 and

275 4). For TIM3, CD27, IDO1 and SDF-1, which are expressed on tumor-associated

276 immune cells, we did not first examine TILs with CD3 staining, as some of the

277 literature has reported. Microscopically, TILs sometimes could be difficult to identify

278 morphologically compared with HE staining, which might also be one of the reasons

279 for the low positive rates observed. Furthermore, a significant proportion of

280 osteosarcoma specimens needed to be decalcified, which also might have resulted in

281 the loss of low molecular expression levels observed in the current study. However,

282 Chen et al. [21] reported that both decalcified and nondecalcified specimens showed

283 evidence of the expression of PD-1 and PD-L1, and decalcification did not cause 284 much change in PD-L1 expression.

$<$ Fig. 3 near here $>$

$<$ Fig. 4 near here $>$

287 At the same time, we performed IHC analyses of the specimens after multiple lines 288 of treatment, which contributed the most to this low positive rate. From our 289 perspectives, chemotherapy for osteosarcoma was so intense that it might 290 tremendously destroy tumor-associated immune cells in the tumor immune 291 microenvironment. It is suspected that these checkpoint molecules might fade away 292 after multiple lines of treatment. Thus, the positive rate might be much lower than that 293 reported by Mochizuki et al. [18], who reported that they investigated the initial 294 treatment samples preserved at diagnosis.

295 On the basis of our experience, only sporadic patients could truly benefit from 
anti-PD-1 therapy, and these patients usually showed evidence of the coexpression of multiple checkpoint molecules. In clinical practice, with so few patients who are responsive to immunotherapy, we usually need to observe patients who are on combination therapy with checkpoint inhibitors, such as chemotherapy or targeted

300 therapy, for longer than a year. In addition, PFS was a more appropriate indicator than 301 the objective response rate for advanced osteosarcoma clinical evaluation because most of our target lesions had calcification and ossification [5]. Although tumor cells sometimes vanished pathologically after treatment, the remains of ossification were still there for clinical evaluation. One of the 5 patients who had evidence of the coexpression of more than one checkpoint molecule had high-intensity staining for 306 B7-H3, CD47 and TGF $\beta$ but was negative for PD-L1 (shown in Fig. 1), however, two 307 years ago, he had high expression of PD-L1 on his tumor cells. The case of this 308 patient demonstrates the evolutionary course of secondary drug resistance to 309 anti-PD-1 antibodies. Later, anti-TGF $\beta$, anti-B7-H3 or anti-CD47 therapy might be 310 practicable for this patient.

311 The limitations of our analysis are that our study was performed at a single 312 institution, utilized a retrospective cohort, and had a relatively small sample size, 313 which precluded us from clarifying whether subtypes with a poor prognosis express 314 checkpoint molecules more frequently than others. Second, we did not collect patient 315 paired samples from biopsy samples before treatment or progressive samples after 316 multiple treatments to observe the evolution of the expression of these checkpoint 317 molecules. Third, we did not examine the expression of tumor-associated checkpoint 318 molecules together with their TILs expressing corresponding receptors for 319 confirmation, which could make the results be more convincing. Fourth, TILs were 320 subjectively identified microscopically by two pathologists individually. The 
321 pathologists did not use CD3 or CD8 staining to identify those TILs, which could 322 make the interpretation difficult and the results unreliable. Finally, the follow-up time 323 for all these patients was not long enough to observe their OS (4/35 (11.4\%) had died 324 from their disease at the time of the last follow-up), which made univariate Cox 325 proportional hazards analysis of OS impossible.

\section{Conclusions}

327 In summary, the present study highlights that only a small subset of progressive 328 osteosarcomas, which had been heavily treated, expressed tumor immune-associated 329 checkpoint molecules. Those osteosarcomas that had ever been responsive to 330 anti-PD-1 therapy usually had evidence of the coexpression of multiple checkpoint 331 molecules, which might also be the reason for secondary drug resistance.

\section{Abbreviations}

333 ABC: Avidin-biotin complex; CDDP: Cisplatin; CEACAM1: Carcinoembryonic 334 antigen-related cell adhesion molecule; CI: Confidence interval; DOX: Doxorubicin; 335 EFS: Event-free survival; EURAMOS-1: European and American Osteosarcoma 336 Study-1; GAL9: Galectin 9; H\&E: Hematoxylin and eosin; $\mathrm{H}_{2} \mathrm{O}_{2}$ : Hydrogen peroxide; 337 HD MTX: High-dose methotrexate; HMGB1: High mobility group protein B1; HR: 338 Hazard ratio; HVEM: Herpesvirus entry mediator; IFO: Ifosfamide; IHC: 339 Immunohistochemical; IQR: Interquartile range; MHC: Major histocompatibility 340 complex; OS: Overall survival; PBS: Phosphate-buffered saline; PD-1: Programmed 341 cell death 1; PD-L1: Programmed cell death 1 ligand 1; PFS: Progression-free 342 survival; PKUPH-OS 02: Peking University People's Hospital-Osteosarcoma; SEM: 343 Standard error of the mean; SHR-1210: Camrelizumab; TCR: T cell receptor; TILs:

344 Tumor-infiltrating lymphocytes; TIM: T cell-immunoglobulin-mucin domain; TKI: 
345 Tyrosine kinase inhibitor; TMA: Tissue microarray; TME: Tumor microenvironment;

346 TNF: Tumor necrosis factor; TNFRSF: Tumor necrosis factor receptor superfamily;

347 UPS: Undifferentiated pleomorphic sarcoma; VCR: Vincristine

348 Supplementary Information

349 Additional file 1: Table S1 Details of the primary antibodies used for 350 immunohistochemistry and antigen retrieval methods (.pdf)

351 Additional file 2: Table S2 Summary of all previous studies for immunochemistry 352 staining of various checkpoint molecules in osteosarcoma specimens (.pdf)

353 Additional file 3: Fig. S1 Pictured is the updated first line osteosarcoma regimen 354 used at Peking University People's Hospital-Osteosarcoma (PKUPH-OS 02). DOX, 355 doxorubicin; CDDP, cisplatin; IFO, ifosfamide; VCR, vincristine; HD MTX, 356 high-dose methotrexate (.pdf)

\section{Declarations}

\section{Ethics approval and consent to participate}

359 Institutional review board approval (No. 2018PHB059-01) to perform this research 360 and review the patients' medical records and radiographic materials was obtained 361 from Peking University People's Hospital. The outcome data were then 362 retrospectively combined. Written informed consent was obtained in Chinese. This 363 study is registered in Clinicaltrials.gov with identifier of NCT03582527 and the first 364 date of registration is July 11th, 2018 (11/07/2018). This study was complied with 365 good clinical practice guidelines and the Declaration of Helsinki.

\section{Consent for publication}

367 Not applicable. 
369 We promised to ensure patients' data confidentially. However, patient data will be 370 made available in a disguised manner, including data dictionaries, for approved data 371 sharing requests. Individual data will be shared that underlie the results reported in 372 this article after the deidentification and normalization of information (text, tables, 373 figures, and supplementaries). The statistical analysis plan will also be available upon 374 request. Anonymized data will be available beginning 3 months later and ending 2 375 years after the publication of this article to researchers after methodological review of 376 the proposed analysis plan by the directors of the Musculoskeletal Tumor Center of

377 Peking University People's Hospital. Proposals should be directed to 378 xie.lu@hotmail.com. To gain access, data requestors will need to sign a data access 379 agreement.

\section{Competing interests}

381 The authors declare no potential conflicts of interest.

\section{Funding}

383 This work was supported by the Beijing Municipal Science \& Technology Project (No. 384 Z181100001718054) and the Research and Development Fund of Peking University 385 People's Hospital (Clinical Medicine + X Cultivation Project, No. RDX2019-08).

\section{Authors' contributions}

387 Conception and design: LX, CLC and WG; Provision of study materials or patients: 388 XL, JX, and XS; Collection and assembly of data: LX, CLC and XL; Pathological 389 review of the slides of this study: KS; Clinical evaluation of the study: RY, XT and 390 WG; Laboratorial work and molecular biological analysis of this study: CLC and XL; 391 Data analysis and interpretation: LX, JX, and WG; Manuscript writing: LX; Final 
approval of the manuscript: LX, CLC, XL, JX, XS, KS, RY, XT and WG;

393 Accountability for all aspects of the work: LX, CLC, XL, JX, XS, KS, RY, XT and 394 WG.

395

396

\section{Acknowledgements}

We thank all the patients and their families for participating in this study. We also thank Dr Yi Ding and Danhua Shen for involvement in the pathological review of all pathological slides. We thank Springer Nature Author Services for its linguistic assistance during the preparation of this manuscript.

\section{References}

1. Baumhoer D, Amary F, Flanagan AM. An update of molecular pathology of bone tumors. Lessons learned from investigating samples by next generation sequencing. Genes Chromosomes Cancer. 2018;58(2):88-99. https://doi.org/10.1002/gcc.22699.

2. Arndt CA, Rose PS, Folpe AL, Laack NN. Common musculoskeletal tumors of childhood and adolescence. Mayo Clin Proc. 2012;87(5):475-87. https://doi.org/10.1016/j.mayocp.2012.01.015.

3. Smeland S, Bielack SS, Whelan J, Bernstein M, Hogendoorn P, Krailo MD, et al. Survival and prognosis with osteosarcoma: outcomes in more than 2000 patients in the EURAMOS-1 (European and American Osteosarcoma Study) cohort. Eur J Cancer. 2019;109:36-50. https://doi.org/10.1016/j.ejca.2018.11.027.

4. Arshi A, Sharim J, Park DY, Park HY, Yazdanshenas H, Bernthal NM, et al. Prognostic determinants and treatment outcomes analysis of osteosarcoma and Ewing sarcoma of the spine. Spine J. 2017;17(5):645-55. https://doi.org/10.1016/j.spinee.2016.11.002. 
416 5. Lagmay JP, Krailo MD, Dang H, Kim A, Hawkins DS, Beaty O, 3rd, et al.

417 Outcome of patients with recurrent osteosarcoma enrolled in seven phase II trials 418 through Children's Cancer Group, Pediatric Oncology Group, and Children's 419 Oncology Group: learning from the past to move forward. J Clin Oncol. $420 \quad$ 2016;34(25):3031-8. https://doi.org/10.1200/JCO.2015.65.5381.

421 6. da Silva JL, Dos Santos ALS, Nunes NCC, de Moraes Lino da Silva F, Ferreira 422 CGM, de Melo AC. Cancer immunotherapy: the art of targeting the tumor 423 immune microenvironment. Cancer Chemother Pharmacol. 2019;84(2):227-40. $424 \quad$ https://doi.org/10.1007/s00280-019-03894-3.

425 7. D'Angelo SP, Mahoney MR, Van Tine BA, Atkins J, Milhem MM, Jahagirdar 426 BN, et al. Nivolumab with or without ipilimumab treatment for metastatic sarcoma (Alliance A091401): two open-label, non-comparative, randomised, phase 2 trials. Lancet Oncol. 2018;19(3):416-26. https://doi.org/10.1016/S1470-2045(18)30006-8.

430 8. Tawbi HA, Burgess M, Bolejack V, Van Tine BA, Schuetze SM, Hu J, et al. 431 Pembrolizumab in advanced soft-tissue sarcoma and bone sarcoma (SARC028): 432 a multicentre, two-cohort, single-arm, open-label, phase 2 trial. Lancet Oncol. 433 2017;18(11):1493-501. https://doi.org/10.1016/S1470-2045(17)30624-1.

434 9. Le Cesne A, Marec-Berard P, Blay JY, Gaspar N, Bertucci F, Penel N, et al. 435 Programmed cell death 1 (PD-1) targeting in patients with advanced 436 osteosarcomas: results from the PEMBROSARC study. Eur J Cancer. 2019;119:151-7. https://doi.org/10.1016/j.ejca.2019.07.018.

438 10. Xie L, Xu J, Sun X, Guo W, Gu J, Liu K, et al. Apatinib plus camrelizumab 439 (anti-PD1 therapy, SHR-1210) for advanced osteosarcoma (APFAO) progressing 440 after chemotherapy: a single-arm, open-label, phase 2 trial. J Immunother Cancer. 
442 11. Keung EZ, Burgess M, Salazar R, Parra ER, Rodrigues-Canales J, Bolejack V, et 443 al. Correlative analyses of the SARC028 trial reveal an association between 444 sarcoma-associated immune infiltrate and response to pembrolizumab. Clin Cancer Res. 2020;26(6):1258-66. https://doi.org/10.1158/1078-0432.CCR-19-1824.

12. Veenstra R, Kostine M, Cleton-Jansen AM, de Miranda NF, Bovee JV. Immune checkpoint inhibitors in sarcomas: in quest of predictive biomarkers. Lab Invest. 2018;98(1):41-50. https://doi.org/10.1038/labinvest.2017.128.

13. Koirala P, Roth ME, Gill J, Piperdi S, Chinai JM, Geller DS, et al. Immune infiltration and PD-L1 expression in the tumor microenvironment are prognostic in osteosarcoma. Sci Rep. 2016;6:30093. https://doi.org/10.1038/srep30093.

14. Chen DS, Mellman I. Oncology meets immunology: the cancer-immunity cycle. Immunity. 2013;39(1):1-10. https://doi.org/10.1016/j.immuni.2013.07.012.

15. Galon J, Bruni D. Approaches to treat immune hot, altered and cold tumours with combination immunotherapies. Nat Rev Drug Discov. 2019;18(3):197-218. https://doi.org/10.1038/s41573-018-0007-y.

458 16. Luo ZW, Liu PP, Wang ZX, Chen CY, Xie H. Macrophages in osteosarcoma immune microenvironment: implications for immunotherapy. Front Oncol.

461 17. Suehara Y, Alex D, Bowman A, Middha S, Zehir A, Chakravarty D, et al. 462 Clinical genomic sequencing of pediatric and adult osteosarcoma reveals distinct molecular subsets with potentially targetable alterations. Clin Cancer Res. 2019;25(21):6346-56. https://doi.org/10.1158/1078-0432.CCR-18-4032.

465 18. Mochizuki K, Kawana S, Yamada S, Muramatsu M, Sano H, Kobayashi S, et al. 
Various checkpoint molecules, and tumor-infiltrating lymphocytes in common pediatric solid tumors: possibilities for novel immunotherapy. Pediatr Hematol Oncol. 2019;36(1):17-27. https://doi.org/10.1080/08880018.2019.1578843.

19. Xu JF, Pan XH, Zhang SJ, Zhao C, Qiu BS, Gu HF, et al. CD47 blockade inhibits tumor progression human osteosarcoma in xenograft models. Oncotarget. 2015;6(27):23662-70. https://doi.org/10.18632/oncotarget.4282.

20. Hashimoto K, Nishimura S, Akagi M. Characterization of PD-1/PD-L1 immune checkpoint expression in osteosarcoma. Diagnostics (Basel). 2020;10(8):528. https://doi.org/10.3390/diagnostics10080528.

21. Chen S, Guenther LM, Aronhalt A, Cardillo L, Janeway KA, Church AJ. PD-1 and PD-L1 expression in osteosarcoma: which specimen to evaluate? J Pediatr Hematol

Oncol.

2020;42(8):482-7. https://doi.org/10.1097/MPH.0000000000001685.

479

22. Huang X, Zhang W, Zhang Z, Shi D, Wu F, Zhong B, et al. Prognostic value of programmed cell death 1 ligand-1 (PD-L1) or PD-1 expression in patients with osteosarcoma: a meta-analysis. J Cancer. 2018;9(14):2525-31. https://doi.org/10.7150/jca.25011.

23. Piperdi S, Roth M, Morriss N, Zinone C, Zhang W, Koirala P, et al. Evaluation of CD47 expression and effects of CD47-SIRP $\alpha$ fusion protein in patients with osteosarcoma. Cancer $\quad$ Res. 2016;76(14 Suppl):2471. https://doi.org/10.1158/1538-7445.AM2016-2471.

24. Xie L, Xu J, Sun X, Li X, Liu K, Liang X, et al. Apatinib plus ifosfamide and etoposide for relapsed or refractory osteosarcoma: a retrospective study in two centres. Oncol Lett. 2021;22(1):552. https://doi.org/10.3892/o1.2021.12813.

25. Xie L, Xu J, Li X, Zhou Z, Zhuang H, Sun X, et al. Complete remission of 
metastatic osteosarcoma using combined modality therapy: a retrospective analysis of unselected patients in China. BMC Cancer. 2021;21:337. https://doi.org/10.1186/s12885-021-08071-5.

494 26. Pinto N, Park JR, Murphy E, Yearley J, McClanahan T, Annamalai L, et al. 495 Patterns of PD-1, PD-L1, and PD-L2 expression in pediatric solid tumors. Pediatr Blood Cancer. 2017;64(11):e26613. https://doi.org/10.1002/pbc.26613. 


\section{Figure legends}

499 Fig. 1 Microscopical manifestation of programmed cell death 1 ligand-1 (PD-L1)

500 staining for advanced osteosarcoma (200×, Abcam 28-8), Expressing PD-L1 in a

$501 \quad$ Membranous Pattern

502 Fig. 2 Microscopical manifestation of one patients' sample with multiple checkpoint 503 modules $(200 \times)$. a High staining Intensity of B7-H3 on tumor cells for his current 504 sample of lymph nodes; b High staining intensity of CD47 on tumor cells for his 505 current sample of lymph nodes; c High staining Intensity of TGFßin diffuse 506 cytoplasmic tumor cells for his current sample of lymph nodes; $\mathbf{d}$ Negative expression 507 of programmed cell death 1 ligand-1 (PD-L1) on tumor cells for his current sample of 508 lymph nodes; e Previous diagnosis with high-grade osteosarcoma in proximal femur 509 with hematoxylin and eosin (H\&E) staining; f Formerly with high staining intensity of 510 PD-L1 (DAKO 22C3) in his amputation specimen; g Current specimen of his 511 metastatic lymph nodes was re-diagnosed with undifferentiated pleomorphic sarcoma $512(\mathrm{H} \& \mathrm{E})$

513 Fig. 3 Negative expression of TIM3 but with background staining in one sample $514(200 \times)$

515 Fig. 4 Negative expression of B7-H3 but with background staining in one sample $516(200 \times)$ 
518 Table 1 Comparison of baseline clinicopathological characteristics based on B7-H3, CD47 and PD-L1 expression in advanced osteosarcoma

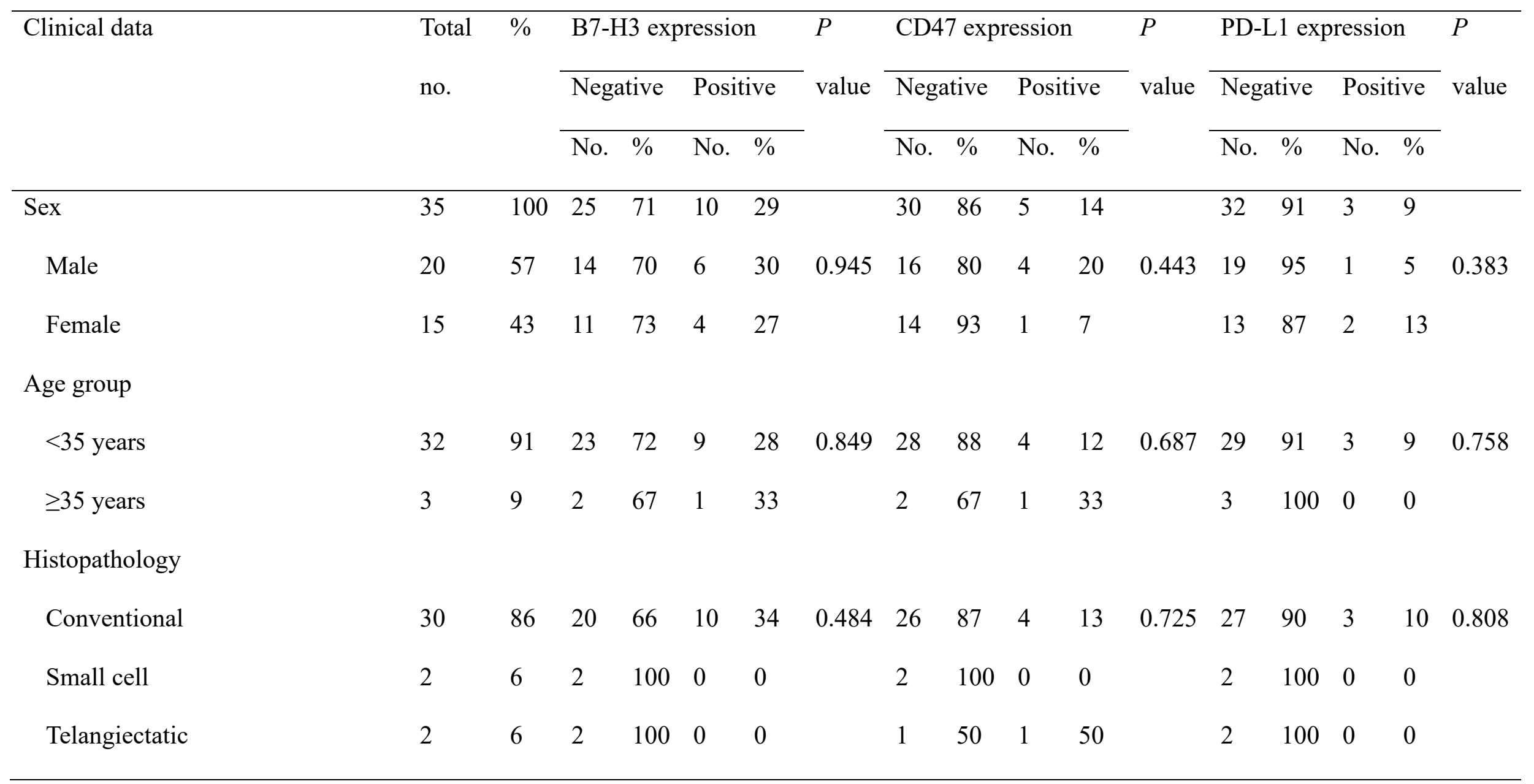




\begin{tabular}{|c|c|c|c|c|c|c|c|c|c|c|c|c|c|c|c|c|c|}
\hline Low grade maltransformation & 1 & 3 & 1 & 100 & 0 & 0 & & 1 & 100 & 0 & 0 & & 1 & 100 & 0 & 0 & \\
\hline \multicolumn{18}{|l|}{ Specimen source } \\
\hline From bone & 25 & 71 & 18 & 72 & 7 & 28 & 0.982 & 21 & 84 & 4 & 16 & 0.258 & 23 & 92 & 2 & 8 & 0.672 \\
\hline From lung & 7 & 20 & 5 & 71 & 2 & 29 & & 7 & 100 & 0 & 0 & & 6 & 86 & 1 & 14 & \\
\hline From lymph nodes & 3 & 9 & 2 & 67 & 1 & 33 & & 2 & 67 & 1 & 33 & & 3 & 100 & 0 & 0 & \\
\hline \multicolumn{18}{|l|}{ Primary tumor site } \\
\hline Distal femur & 15 & 43 & 9 & 60 & 6 & 40 & 0.084 & 12 & 80 & 3 & 20 & 0.079 & 14 & 93 & 1 & 7 & 0.761 \\
\hline Proximal tibia & 8 & 23 & 6 & 75 & 2 & 25 & & 8 & 100 & 0 & 0 & & 7 & 88 & 1 & 12 & \\
\hline Proximal humerus & 4 & 11 & 4 & 100 & 0 & 0 & & 4 & 100 & 0 & 0 & & 3 & 75 & 1 & 25 & \\
\hline Proximal femur & 1 & 3 & 0 & 0 & 1 & 100 & & 0 & 0 & 1 & 100 & & 1 & 100 & 0 & 0 & \\
\hline Axial skeleton & 6 & 17 & 5 & 83 & 1 & 17 & & 6 & 100 & 0 & 0 & & 6 & 100 & 0 & 0 & \\
\hline Others & 1 & 3 & 1 & 100 & 0 & 0 & & 0 & 0 & 1 & 100 & & 1 & 100 & 0 & 0 & \\
\hline \multicolumn{18}{|l|}{ Chemotherapy resistance } \\
\hline Yes & 14 & 40 & 11 & 79 & 3 & 21 & 0.445 & 10 & 71 & 4 & 29 & 0.019 & 14 & 100 & 0 & 0 & 0.259 \\
\hline No & 21 & 60 & 14 & 67 & 7 & 33 & & 20 & 95 & 1 & 5 & & 18 & 86 & 3 & 14 & \\
\hline
\end{tabular}




\begin{tabular}{|c|c|c|c|c|c|c|c|c|c|c|c|c|c|c|c|c|c|}
\hline \multicolumn{18}{|l|}{ Anti-angiogenesis TKI resistance } \\
\hline Yes & 4 & 11 & 3 & 75 & 1 & 25 & 0.867 & 2 & 50 & 2 & 50 & 0.365 & 4 & 100 & 0 & 0 & 0.687 \\
\hline No & 31 & 89 & 22 & 71 & 9 & 29 & & 28 & 90 & 3 & 10 & & 28 & 90 & 3 & 10 & \\
\hline \multicolumn{18}{|l|}{ Anti-PD-1 therapy efficacy } \\
\hline Effective & 4 & 11 & 2 & 50 & 2 & 50 & 0.057 & 3 & 75 & 1 & 25 & 0.061 & 4 & 100 & 0 & 0 & 0.105 \\
\hline Invalid & 9 & 26 & 9 & 100 & 0 & 0 & & 8 & 89 & 1 & 11 & & 9 & 100 & 0 & 0 & \\
\hline Unused & 17 & 49 & 11 & 65 & 6 & 35 & & 16 & 94 & 1 & 6 & & 16 & 94 & 1 & 6 & \\
\hline $\begin{array}{l}\text { Not determinable (combination } \\
\text { therapy) }\end{array}$ & 5 & 14 & 3 & 60 & 2 & 40 & & 3 & 60 & 2 & 40 & & 3 & 60 & 2 & 40 & \\
\hline
\end{tabular}


521 Table 2 Scoring of various checkpoint molecule immunochemistry staining intensities for 35 advanced osteosarcomas $\dagger$

\begin{tabular}{|c|c|c|c|c|}
\hline Checkpoint molecules & Positivity & \multicolumn{3}{|c|}{ Staining intensity } \\
\hline CD47 & $5 / 35(15 \%)$ & $2 / 35(6 \%)$ & $2 / 35(6 \%)$ & $1 / 35(3 \%)$ \\
\hline TIM3 & $2 / 35(6 \%)$ & $2 / 35(6 \%)$ & $0 / 35(0 \%)$ & $0 / 35(0 \%)$ \\
\hline TGF $\beta$ & $2 / 35(6 \%)$ & $0 / 35(0 \%)$ & $1 / 35(3 \%)$ & $1 / 35(3 \%)$ \\
\hline
\end{tabular}

522 †If these checkpoint molecules were expressed on the tumor cells, a semiquantitative scoring system $(0-<1 \%=$ negative $/$ rare, $1-<10 \%=10 w$,

$52310-<50 \%=$ moderate, and $>50 \%=$ high) was applied for the evaluation of immune checkpoint ligand expression; If these checkpoint molecules

524 were expressed on the tumor-infiltrating lymphocytes, a semiquantitative scoring system $(0-<1 \%=$ negative $/$ rare, $1-<10 \%=10$, $10-<50 \%=$

525 moderate, and $>50 \%=$ high) was applied for the evaluation of immune checkpoint receptor expression on the surface of the CD3-positive

526 tumor-infiltrating lymphocytes (TILs). 
Table 3 Univariate Cox proportional hazards analysis of PFS and OS for patients with advanced osteosarcoma

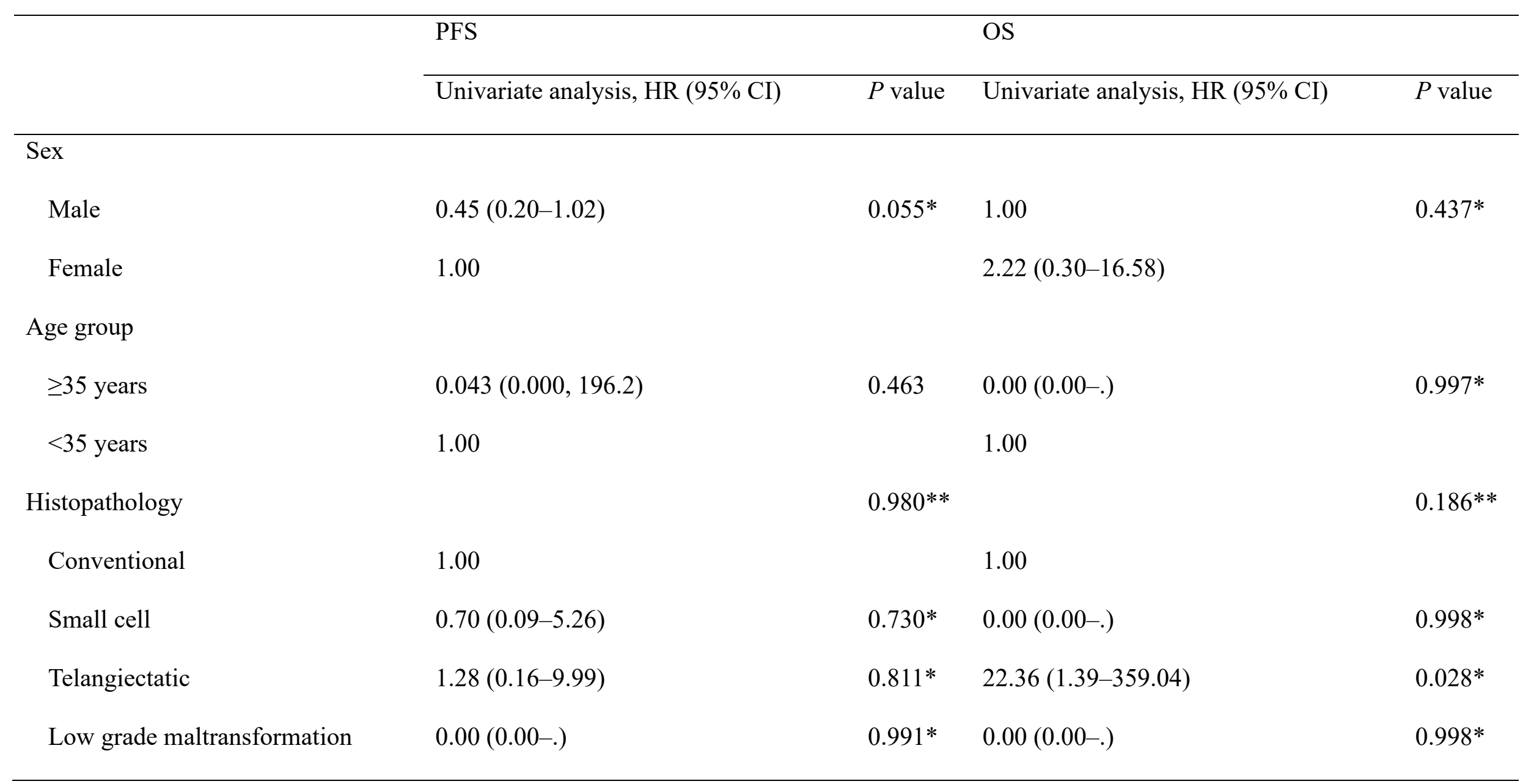




\begin{tabular}{|c|c|c|c|c|}
\hline Primary tumor site & & $0.907 * *$ & & $0.989 * *$ \\
\hline Proximal humerus & $1.15(0.32-4.19)$ & $0.829 *$ & $0.00(0.00-)$. & $0.998^{*}$ \\
\hline Proximal femur & $1.45(0.18-11.62)$ & $0.725^{*}$ & $0.00(0.00-)$. & $0.999 *$ \\
\hline Axial skeleton & $0.52(0.14-1.87)$ & $0.314^{*}$ & $1.76(0.25-12.58)$ & $0.573^{*}$ \\
\hline Others & $0.00(0.00-)$. & $0.994 *$ & $0.00(0.00-)$. & $0.999 *$ \\
\hline B7-H3 & & $0.317^{*}$ & & $0.760 *$ \\
\hline Positive & $1.61(0.63-4.08)$ & & $1.43(0.14-14.21)$ & \\
\hline Positive & $1.63(0.55-4.83)$ & & $0.00(0.00-)$. & \\
\hline Negative & 1.00 & & 1.00 & \\
\hline PD-L1 & & $0.858^{*}$ & & $0.217^{*}$ \\
\hline Positive & $0.88(0.20-3.78)$ & & $4.55(0.41-50.46)$ & \\
\hline
\end{tabular}




\begin{tabular}{|c|c|c|c|c|}
\hline Negative & 1.00 & & 1.00 & \\
\hline 1 checkpoint & $1.28(0.45-3.60)$ & $0.642 *$ & $1.20(0.12-11.53)$ & $0.877^{*}$ \\
\hline Negative for all & 1.00 & & 1.00 & \\
\hline At least one positive & $1.06(0.45-2.49)$ & & $0.71(0.07-6.97)$ & \\
\hline All negative & 1.00 & & 1.00 & \\
\hline
\end{tabular}

529 CI, confidence interval; HR, hazard ratio; OS, overall survival; PD-L1, programmed cell death 1 ligand 1; PFS, progression-free survival.

$530 *$ Covariate Wald $P$-value; **Type 3 Wald $P$-value. 


\section{Figures}

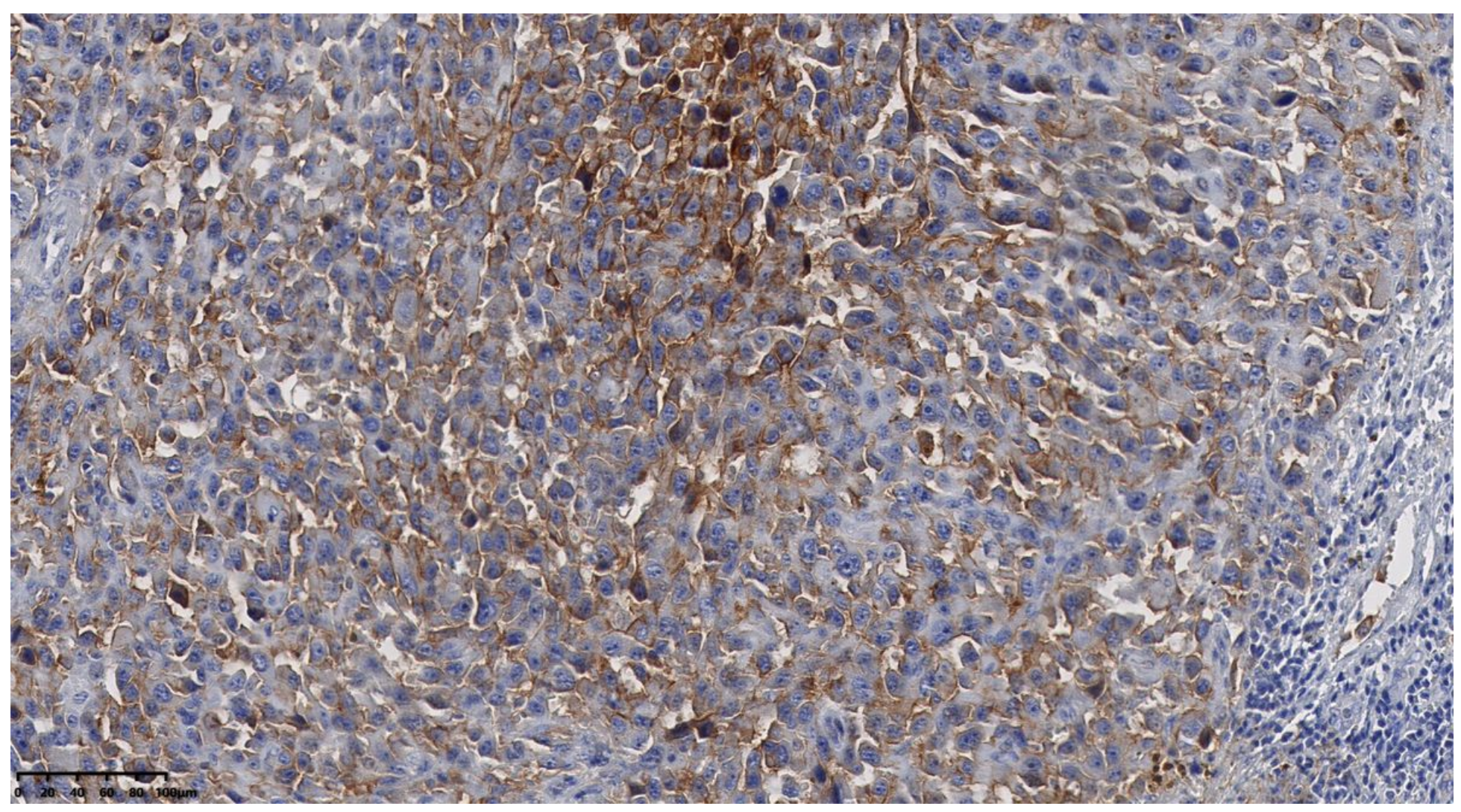

\section{Figure 1}

Microscopical manifestation of programmed cell death 1 ligand-1 (PD-L1) staining for advanced osteosarcoma (200×, Abcam 28-8), Expressing PD-L1 in a Membranous Pattern 

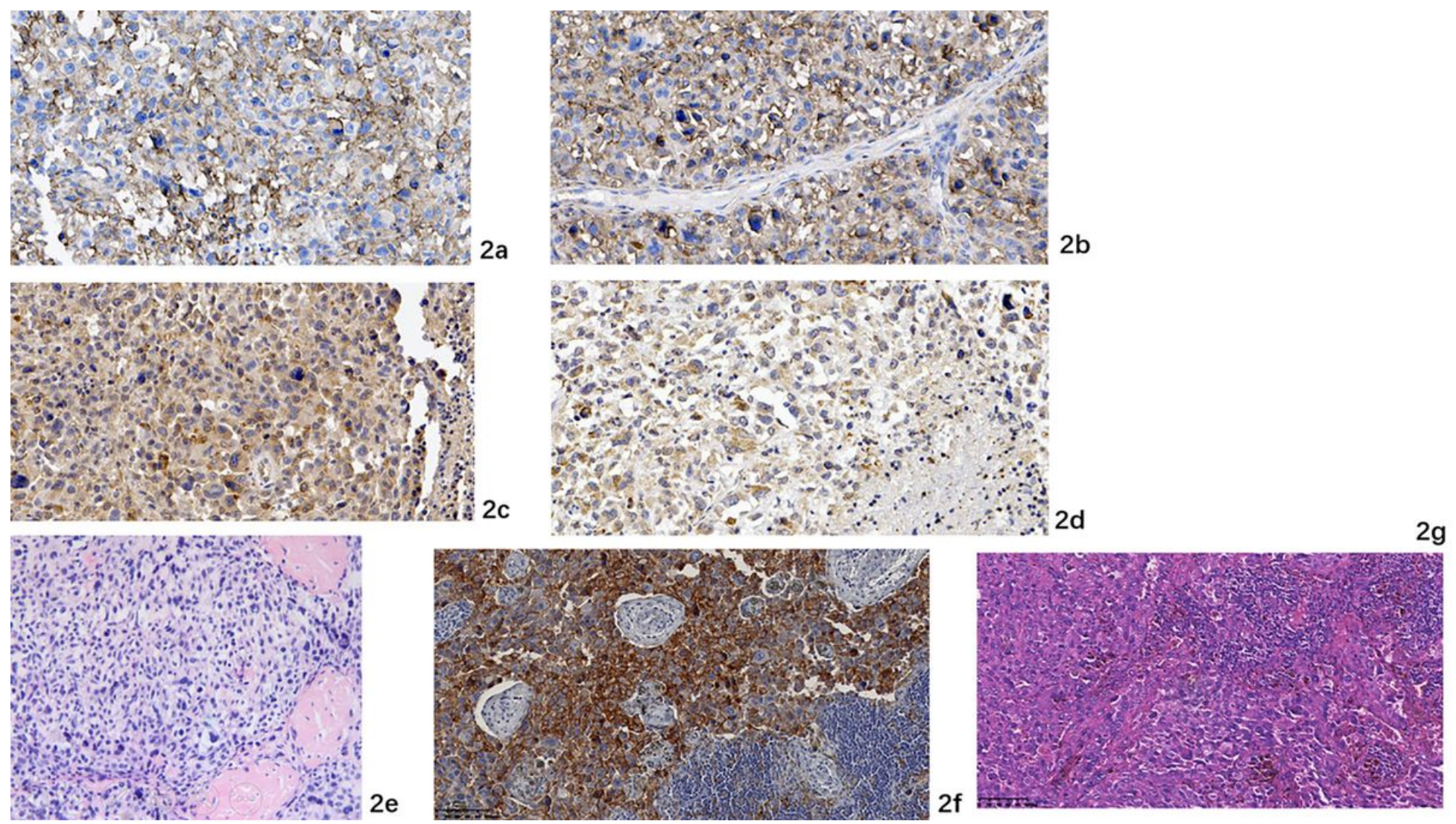

\section{Figure 2}

Microscopical manifestation of one patients' sample with multiple checkpoint modules (200x). a High staining Intensity of B7-H3 on tumor cells for his current sample of lymph nodes; b High staining intensity of CD47 on tumor cells for his current sample of lymph nodes; c High staining Intensity of TGFßin diffuse cytoplasmic tumor cells for his current sample of lymph nodes; d Negative expression of programmed cell death 1 ligand-1 (PD-L1) on tumor cells for his current sample of lymph nodes; e Previous diagnosis with high-grade osteosarcoma in proximal femur with hematoxylin and eosin (H\&E) staining; f Formerly with high staining intensity of PD-L1 (DAKO 22C3) in his amputation specimen; $g$ Current specimen of his metastatic lymph nodes was re-diagnosed with undifferentiated pleomorphic sarcoma (H\&E) 


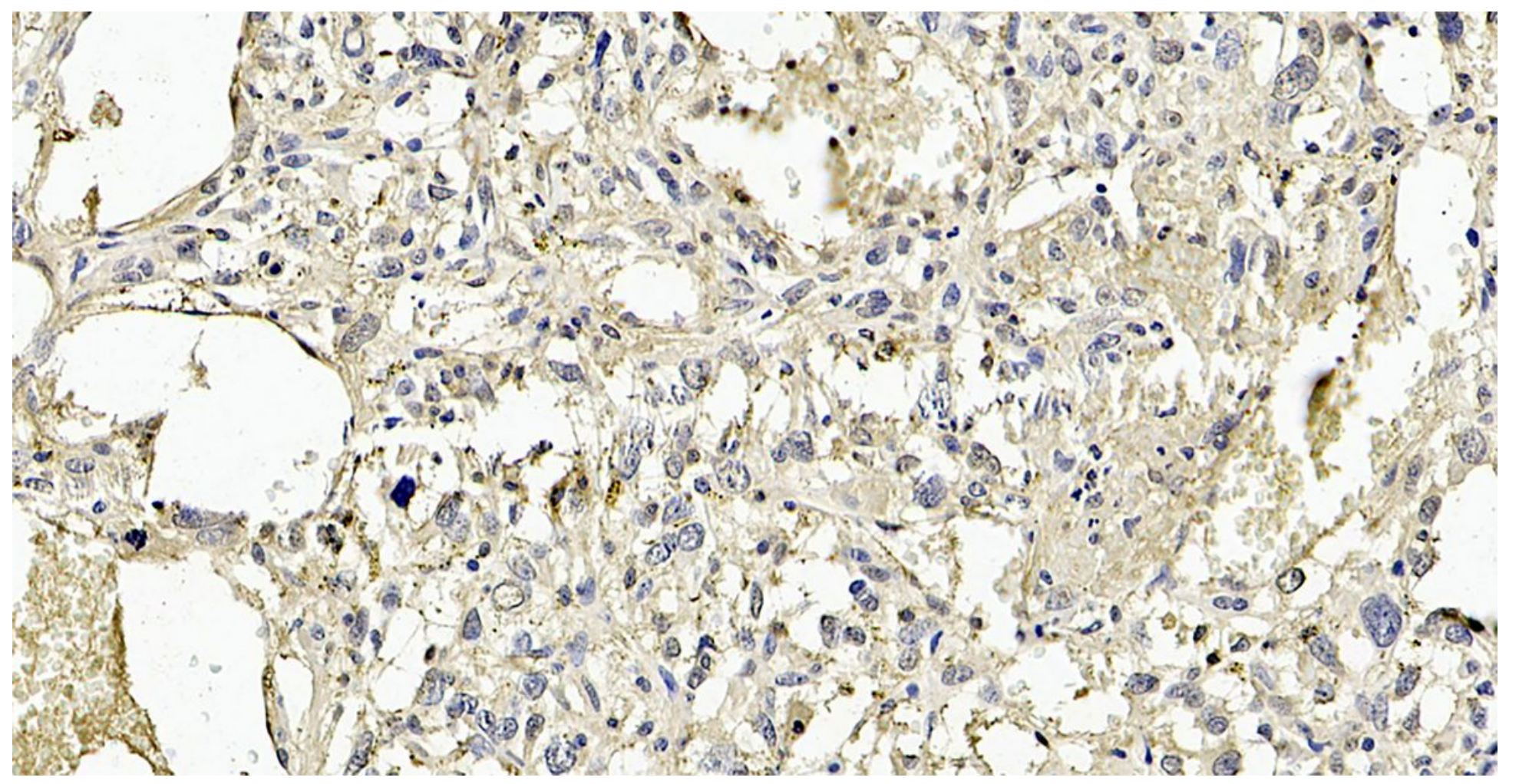

Figure 3

Negative expression of TIM3 but with background staining in one sample (200x)

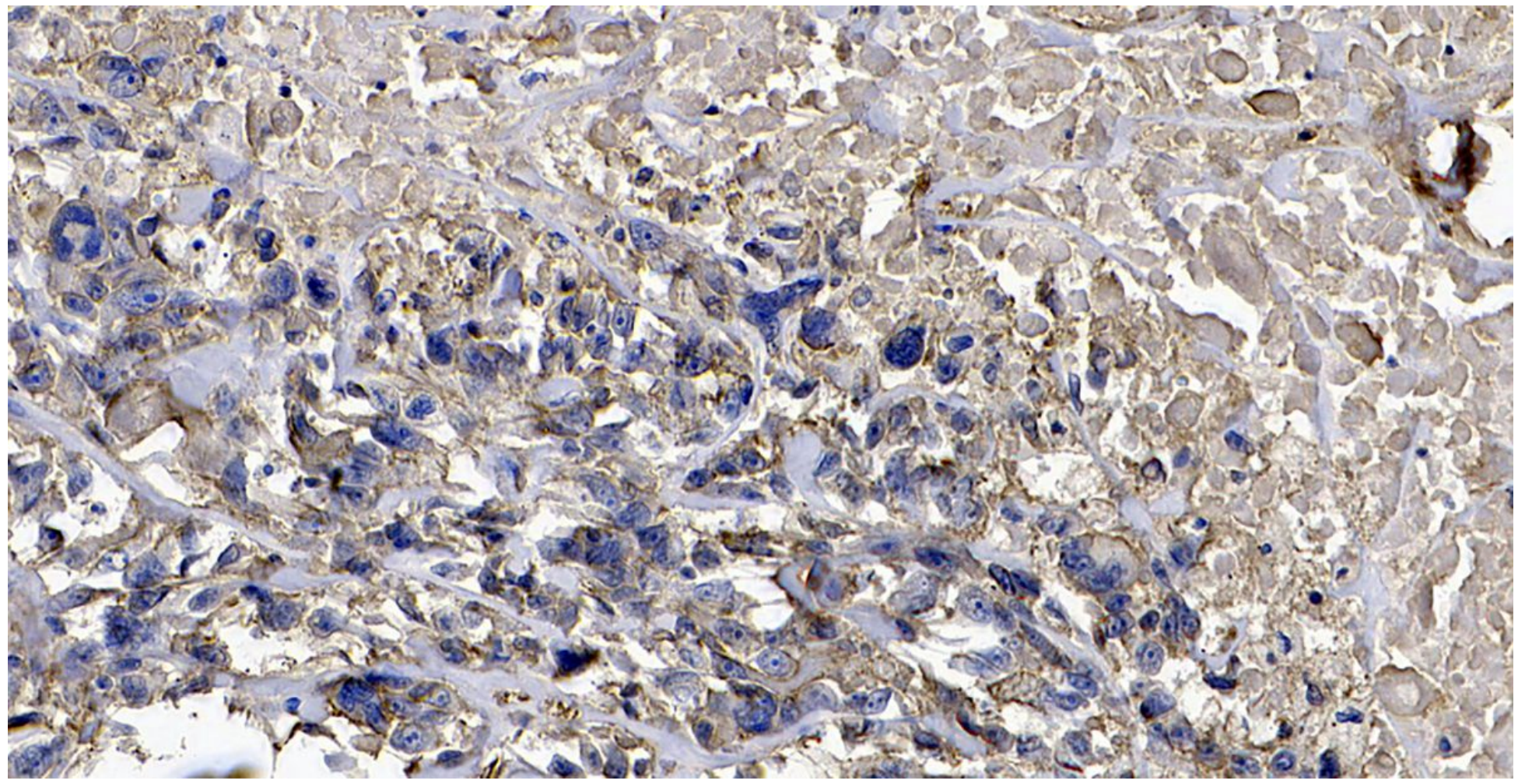

Figure 4

Negative expression of $\mathrm{B} 7-\mathrm{H} 3$ but with background staining in one sample(200x) 


\section{Supplementary Files}

This is a list of supplementary files associated with this preprint. Click to download.

- Additionalfilesvfinal.pdf 